\title{
Review of the Cooling Technology for High-power Wind Turbines
}

\author{
Sheng Jian ${ }^{\mathrm{a}}$, Meng Xiaoqian ${ }^{\mathrm{b}}$, Chu Shuying $^{\mathrm{c}}$ and Guo Huijing ${ }^{\mathrm{d}}$ \\ School of Energy and Power Engineering, University of Shanghai for Science and Technology , \\ 516 Jungong Road, Yangpu District, Shanghai, China, 200093

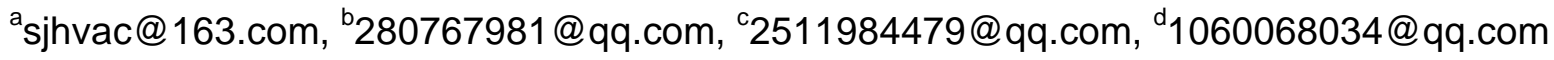

Keywords: wind turbine; cooling technology; heat pipe

Abstract. With the increasing of wind turbine unit capacity, heat load of thermal dissipation components of the unit increase greatly. In order to make the unit long-term stably and efficiency operation, the research and development of cooling system with high efficiency and low energy consumption become particularly important. The main sources of waste heat and the widely used six kinds of cooling technology was described. Also there is a detailed analysis of the pros and cons of each one, and the gravity heat pipe was designed which is instead of the above cooling systems. This cooling technology have high heat transfer efficiency and no cooling medium delivery device and simple structure and low initial investment and operating costs.

\section{Introduction}

According to the Global Wind Energy Council statistics, 2014 global new capacity is $51477 \mathrm{MW}$, and Chinese new capacity is $23351 \mathrm{MW}, 45.2 \%$ of the total. The global cumulative installed capacity is $369553 \mathrm{MW}$ in 2014 , while the cumulative installed capacity is $114763 \mathrm{MW}$ in China, $31.0 \%$ of the total, ranking the first of the world. At present, $6 \mathrm{MW}$ offshore wind turbine has been developed and produced by Guodian United Power Technology (Baoding) Co., Ltd. which is the single most powerful wind turbine currently. Due to the rapid growth in unit capacity, the waste heat of wind turbine increases a lot. A more efficient cooling system is needed to timely discharge the heat to the environment, which is extremely important for a long-term stable operation of wind turbines.

Currently, researchers focused on seismic measures, inverter technology and mechanical design and other fields in China, but the cooling technology of wind turbine is rarely researched ${ }^{[1]}$. In foreign, the introduction of high-power wind turbines are rarely reported due to technical protection. The present situation of cooling technology for wind turbine are summarized, and several kinds of typical cooling technology are comprehensive compared and a more energy efficient new wind turbine cooling system is designed.

\section{The heat load on the wind turbine}

The wind turbine generates electricity by wind, but about $5 \%$ power is changed to waste heat and the main heat-generating components include gear box, control inverter, generator, brake mechanism, direction adjust device and variable pitch system, etc. The heat load is related to the wind turbine's type, size and production process, etc. And in the MW wind turbines, the gear box, generator and control inverter are the main heating components.

The waste heat produced by gear box. The heat load in the gear box mainly raises the oil temperature. The heat is mainly produced by gear box process to overcome the friction that part of kinetic energy change into heat energy, which causing temperature rise ${ }^{[2]}$. The viscosity of the oil will decline and become thin by the temperature increase, so lubricating oil film is destroyed, the gear's friction increases, and calorific value increase more, which resulting in a vicious cycle. And if the temperature is too high that makes the tooth surface gluing, so as to damage the gear, which may result in huge losses. Therefore, to ensure the normal operation, heat exchange between the gear box and cooling fluid must be good ${ }^{[3]}$. 
The waste heat produced by generator. The heat load of the generator comes from: (1) Copper loss, namely generator components around the winding copper wires, due to the copper wire resistance, part of electrical energy is transformed into heat energy; (2) Iron loss, is due to the rotation of the core hysteresis effect of eddy current, resulting in hysteresis loss and eddy current loss; (3) Mechanical loss, that part of the energy is used during generator operation to overcome the friction. All of these energy loss will ultimately transformed into heat energy, distributed into the wind turbine nacelle ${ }^{[4]}$. The linear generator size, magnetic load and line load are the main ways to increase unit capacity. But increasing the linear dimension of generator, the losses will increase with the generator proportional to the cube of linear dimensions, resulting in a serious decline in generator efficiency. Therefore, reducing the temperature rise is very important ${ }^{[5 \sim 7]}$.

The waste heat produced by control inverter. The heat of control inverter mainly comes from the electronic component with impedance, such as capacitors, thyristor, SCR. When the current through the impedance will convert part of the electrical energy into heat energy. Inverter prone to failure by power unit over-temperature, such as the control inverter working environment temperature increased every $10^{\circ} \mathrm{C}$, will make control inverter life reduced by half, while greatly increasing failure rate. Therefore, the heat problem of inverter determines that inverter is working properly, in order to prevent temperature of the various components of the power unit is too high ${ }^{[8]}$.

\section{The cooling technology of wind turbine}

Wind turbine cooling technology can be divided for air cooling system and liquid cooling system. And air cooling system can be divided into natural ventilation cooling and forced air cooling. According to statistics, about $95 \%$ of the wind turbine using forced air cooling and liquid cooling ${ }^{[9]}$. The cooling technology commonly used at present in Tab. $1^{[10]}$.

Table 1 Comparison of several cooling technology

\begin{tabular}{ccccccc}
\hline Cooling technology & Cooling medium & Volume & Cost & $\begin{array}{c}\text { Cooling } \\
\text { capacity }\end{array}$ & $\begin{array}{c}\text { Service } \\
\text { life }\end{array}$ & $\begin{array}{c}\text { Cooling } \\
\text { efficiency }\end{array}$ \\
\hline air-air & air & big & low & small & short & low \\
liquid-air & lubricating oil & small & low & medium & medium & $\begin{array}{c}\text { medium } \\
\text { high }\end{array}$ \\
air-liquid-air & antifreeze & medium & medium & big & medium & high \\
liquid-liquid-air & antifreeze & medium & medium & big & medium & high \\
air-liquid-liquid & antifreeze & medium & medium & big & medium & high \\
centralized cooling & Water or air & big & high & big & long & medium \\
\hline
\end{tabular}

Air-air cooling technology. Air-air cooling technology is the outside cold air exchange heat with the hot air by natural air convection or forced air convection so as to cool the components.

No cooling equipment is add in nature ventilation cooling system which the cold air outside and heated air inside exchanges heat by natural convection. Due to natural convection heat transfer coefficient is small, so it is only suitable for low-power wind turbines.

Forced air cooling uses forced air convection, installing a blower or fan inside of the wind turbine, when the air passing the heat surface at a high speed, can destroy the boundary layer and reduce the thermal resistance and increase the convective heat transfer coefficient ${ }^{[8]}$. Generally, the unit capacity below $300 \mathrm{~kW}$, generator and control inverter has small amount of heat dissipation, heat mainly concentrated on the gear box. For generators, cooling fans can be set internal and external. For control inverter, cooling fans also be set in the inverter motor tail or the control cabinet outlet. And a fan is mounted in the high-speed shaft in the gear box, through the gear box shaft rotation drives fan to deliver cold air ${ }^{[3]}$. In theory, the more air volume and the faster air velocity, the better cooling effect, but the oversized cooling fan will cause generator wind friction loss increases, reducing the efficiency of the generator.

Air-air cooling technology $\mathrm{m}$ is simple, the initial investment and operating costs are low, and easy to maintenance and management. But the cooling effect is influenced by the external environment, such as temperature, weather change, etc., with big restriction and small cooling capacity. Air circulation 
process will cause the corrosion problem of the cabin interior equipment, as well as by the impact of space heat and temperature distribution is not uniform and cause material deformation ${ }^{[11]}$.

Liquid-air cooling technology. The waste heat transfers to circulate liquid coolant first in liquid-air cooling technology, then exchanges heat with the outside high speed forced convection air in a heat exchanger, after the coolant cools down and back to the internal equipment. Now most gear boxes use liquid-air cooling technology. When the capacity is more than $300 \mathrm{~kW}$, power dissipation greatly increases in gear box so as to forced air cooling cannot solve waste heat problem, and the only way is the lubricating oil circulating system which has a better cooling capacity. When the turbine runs at high speed, the heat will be taken away by the lubricating oil which pressurized by a mechanical pump into the heat exchanger, after the high speed air flowing through the other side of the heat exchanger to cool the oil that will back to the gear box (Fig.1). And it is different from the finned tube heat exchanger used by air-air cooling, to enhance heat transfer effect, aluminum plate-fin heat exchanger is widely used, which has small volume, light weight, high thermal efficiency, strong adaptability, and the heat transfer capacity of per unit volume is ten times than finned twhe heat exchanoer

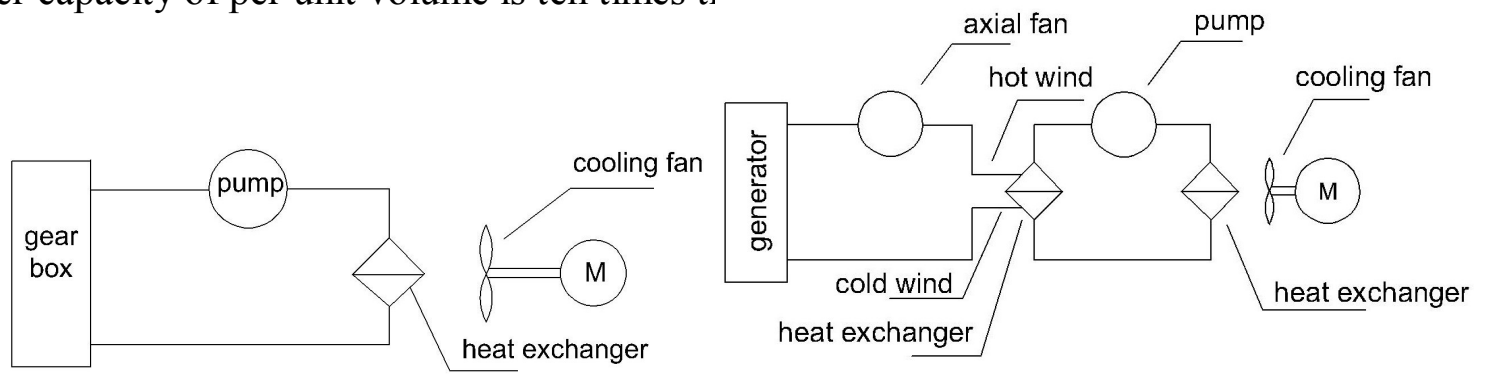

Fig.1 Liquid-air cooling technology Fig.2 Air-liquid-air cooling technology

Liquid-air cooling technology uses in high capacity wind turbine, whose structure is more complex. Due to the increase of the heat exchanger and the coolant, it rises the initial investment and maintenance costs. But its cooling effect improves significantly. Although the main components of the wind and rain erosion solves, but the corrosion problem of the heat exchanger comes. Due to plate-fin heat exchanger small volume and high efficiency, it can effectively solve the uneven distribution of heat and temperature problems, and reduce wind abrasion and improve efficiency.

Air-liquid-air cooling technology. As the capacity of wind turbine is increasing, air-air cooling technology and liquid-air cooling technology are not enough to cooling load. Therefore higher heat transfer efficiency of air-liquid-air cooling technology is introduced. Air-liquid-air cooling technology means that the heat generated by the device is cooled by the coolant through two heat exchangers. For example, hot air in generator first exchanges heat with the circulating coolant $\mathrm{A}$ in the first heat exchanger, then the circulating coolant $\mathrm{B}$ with large heat flows to the second heat exchanger outside the engine cabin, and exchanges heat with external high-speed flow air by forced convection. After cooling, the coolants back to the generator to continue the next cycle (Fig.2). Now commonly used as coolant is antifreeze, mainly glycol water solution. The addition of stabilizers and preservatives make the coolant has a close thermal performance as water, but also has better freezing properties, when the volume concentration of $60 \%$, with the lowest freezing point $-50.1^{\circ} \mathrm{C}$, which can meet the requirements of low-temperature unit ${ }^{[12]}$.

Air-liquid-air cooling technology adds a cooling system and makes the structure more complex. Also the initial investment and maintenance costs significantly increased. But it has greater cooling capacity and efficiency. So no sand and water erosion problem, it provides the wind turbine a favorable working environment and extend its life. The structure is complex, but more compact, and a moderate volume between air-air cooling and liquid-air cooling.

Liquid-liquid-air cooling technology. Liquid-liquid-air cooling technology and air-liquid-air cooling technology are similar. Also heat is exchanged by the circulating coolant in two runs, except liquid instead of air in the first run. For MW wind turbine, the waste heat of gear box surges, even liquid-air cooling is not enough. Then it is cooled by liquid-liquid-air that provides better heat transfer. Hot lubricating oil is pushed into the first liquid-liquid heat exchanger by mechanical pump, and cooled by 
intermediate coolant. Then the intermediate coolant is circulated to the liquid-air heat exchanger which is cooled by the high-speed air by forced convection (Fig.3). Because it is similar to the air-liquid-air cooling, whose coolant is antifreeze, glycol water solution is often used.

Liquid-liquid-air cooling technology is suitable for wind turbines with high-power gear box. Its structure similar as air-liquid-air cooling, but the initial investment and maintenance increased. However, heat change mediums in the first heat exchanger are both liquid, so the heat transfer coefficient of liquid and liquid is much greater than air and liquid, therefore a large cooling capacity. There is no sand and water erosion problems, so the equipment life will extend effectively.

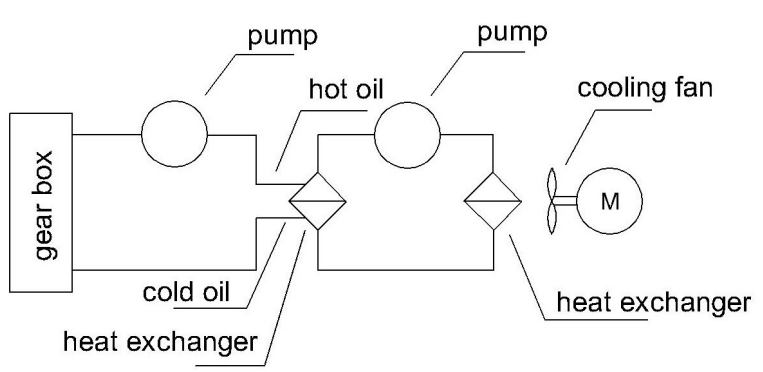

Fig.3 Liquid-liquid-air cooling technology

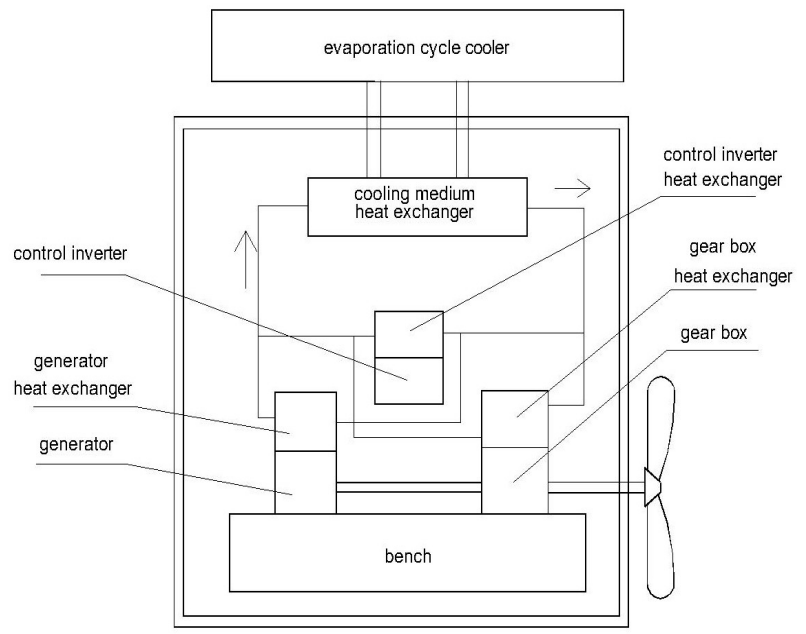

Fig.4 Evaporation cycle cooling technology Air-liquid-air cooling technology. Air-liquid-liquid cooling technology is also known as evaporation cooling cycle technology ${ }^{[13]}$. And an evaporation cycle cooler is set in the extravehicular of wind turbines that intermediate circulating coolant is no longer simply using forced air, but by the special arrangement of the cooling system for cooling through evaporation and condensation processes. Evaporation cycle cooler may be disposed in the rear cabin, also can be placed on the top of the nacelle to reduce its weight ${ }^{[14]}$, as shown in Fig.4.

Air-liquid-liquid cooling technology is used in high-power wind turbine. The evaporation cycle refrigerator cooling effect is better, but the refrigerator will increase the cost of the initial investment and the operating power is far greater. Thereby it has a worse power generation efficiency. To enhance the effect of heat transfer, the refrigerator should be placed outside the nacelle, so wind and rain erosion problems inevitably, but require regular maintenance, and the life is short.

Centralized cooling technology. Centralized cooling technology is different from the single cooling unit, which has a center cooling unit in the whole wind farm to provide cooling capacity for each wind turbine. The heat of gear box, generator and control inverter is still taken away by their external heat exchanger with the refrigerant. Then the coolant which has a higher temperature flows back to the center cooling unit to reduce the temperature. After cooling to designed temperature, the coolant is delivered by circulating pump to each unit. To avoid sand and rain erosion, the coolant and the recycling pipeline are generally fixed together with the electric cable in the tower interior (Fig.5). The coolant can be water or air. But the height of the tower is often ten meters to hundred meters, if water is chose as the coolant, although thermal effect is better, but more power is needed to pump it. Both from the economic and energy utilization aspects, it produces huge energy losses ${ }^{[15]}$.

Centralized cooling technology can be regulated according to cooling capacity changes by equipment requirements and seasons. So it has strong flexibility and wide range. However, this cooling system needs long pipelines laid in the wind farm, not only refrigerant and pipe material cost more, but also the energy consumption of pump is huge and maintenance is difficult. 


\section{The new wind turbine cooling technology}

Throughout the above cooling technologies, there are some problems. Due to the increased unit capacity, cooling equipment not only makes the efficiency decreased and costs increased, but also brings corrosion problems. In recent years, heat pipe cooling technology is intensive researched, which has excellent thermal conduction, heat flux variability, reversible flow direction, constant temperature properties, good environment adaptability, compact structure and low cost, etc.. If heat pipe is used to instead of the usual heat exchanger, not only high heat transfer rate can be required, but also corrosion problems and initial investment and maintenance cost will be effectively reduced.

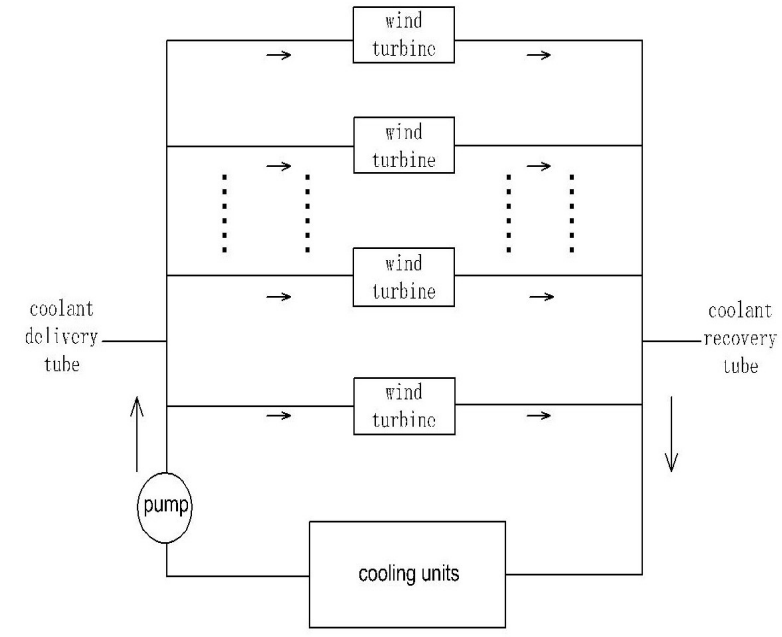

Fig.5 Centralized cooling technology

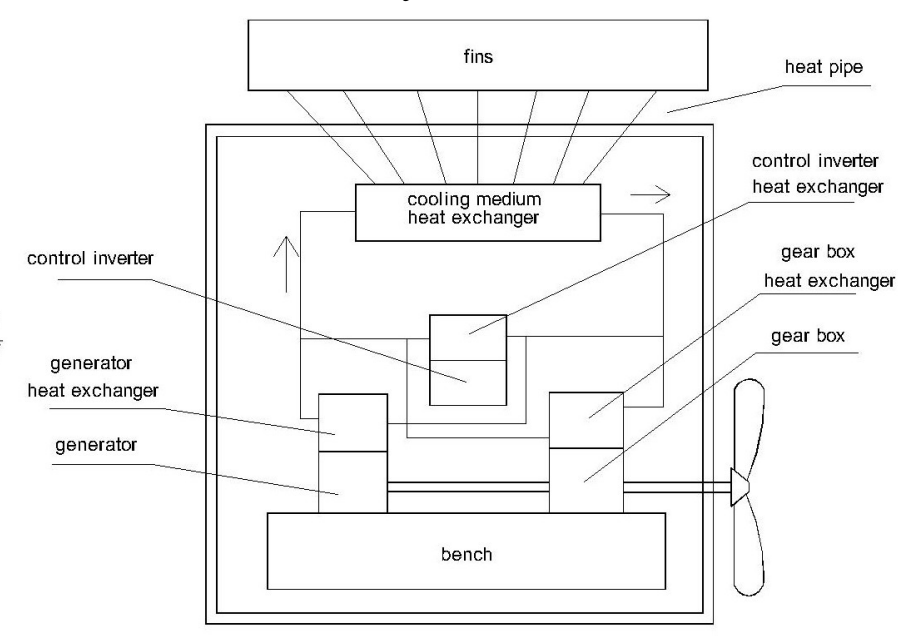

Fig.6 Heat pipe cooling technology

The heat pipe is made up of shell and wick and end cap. A negative pressure within the pipe which is filled with a proper amount of working medium. Heat pipe is divided into three sections: the evaporator section, adiabatic section and condenser section. The working medium is evaporated by hot resource in the evaporator section, thus there is a slight pressure difference at both ends. Working medium (gas) will pass through adiabatic section to condenser section with lots of heat by the pressure difference. Then the gas condensates to liquid in the condenser section and conducts heat to environment. Finally the liquid will flow back to the evaporator section by gravity. Heat pipe transfers heat with the phase change and its thermal capacity is more than any known metal, which has a very high heat transfer efficiency and excellent cooling effect ${ }^{[8,16]}$. Many substances can be used as working medium in heat pipe, such as water, ammonia, methanol, acetone, R11, R113 and so on. So the most widely used is water, the scope of work is $30 \sim 200^{\circ} \mathrm{C}^{[17]}$. Therefore, improvements are made as follows: intermediate cooling medium is cooled by heat pipe, this can increases the efficiency of cooling and reducing the cost and size of the volume (Fig.6).

New cooling system is not only suitable for high-capacity wind turbine, but also its structure is greatly simplified, so its size and maintenance and operation cost. This system has low energy consumption and high efficiency.

\section{Conclusion}

The continuous development of high-power wind turbine makes the unit capacity of wind turbines increasing a lot, but this brings challenges to the cooling technology. The current advantages and disadvantages of the commonly used cooling technologies are summarized. So the new cooling technology with gravity heat pipe has designed to instead. The new technology has higher heat transfer efficiency, simpler structure, lower initial and operation costs. So it will be widely used in wind turbine cooling system. 


\section{Acknowledgement}

The authors gratefully acknowledge the support by Teaching Reform Project (2015-JPBKY-030) of University of Shanghai for Science and Technology.

\section{References}

[1] Weiwei Yuan, Study on the cooling system of wind power generation, Nanjing: Nanjing University of Aeronautics and Astronautics, 2008.

[2] Jian Zhang, Ruiqing Liu, Discuss of the gear box of wind turbine generator, Wind power, 2004, 72(1): 17-20.

[3] Daguang Wang. Thermal power calculation of gearbox for electric speed, Wind power, 2003, 15(3): 22-24.

[4] Shunnian Ding, Heating and cooling of large motor, Science Press, Beijing, 1992.

[5] Yunqiu Tang, Nai Shi, Electrical machinery, China Machine Press, Beijing, 2002.

[6] Tianjun Ni, Main Cooling mode and characteristics of large generator. Dongfang Electric Review, 2006, 20(1): 31-37.

[7] Tskhvirashvili D.G., Berishvili Z.D., Keshelava V.G., Tsagareli K., Experience in operating the water-cooling system of the generator stator windings at the inguri hydroelectric station, Power Technology and Engineering (Formerly Hydro Technical Construction), 1990, 24(8):23-24.

[8] Helun Dong, Choice and performance of high-voltage inverter radiator, Fuxin: Liaoning University of Engineering and Technology, 2011.

[9] Zhou Dao, Frede Blaabjerg, Mogens Lau, Michael Tonnes, Thermal profile analysis of double-fed induction generator based wind power converter with air and liquid cooling methods, Power Electronics and Applications, 2013, 15:1-10.

[10] Bin Yuan, Yuhong Shen, Duo Zong, Research on cooling systems in high-power wind turbine, Dongfang Turbine, 2012, 4:6-9.

[11] Zhili Liu, Design and experimental study of large-scale wind power generator cooling system, Ningjing: Nanjing University of Aeronautics and Astronautics, 2012.

[12] Zhicheng Tan, Glycol and water binary system of physical and chemical properties data, Chemical Engineering (China), 1983(1): 41-50.

[13] Yanlong Jiang, Weiwei Yuan, Hualin Li, Wind turbine generator with evaporative cooling system. Patent application No.200610039658.9.

[14] Wang Li, Haifeng Wang, Steady-state thermal simulation of the stator coil of the evaporative inner cooling system in wind turbines, Information and Automation for Sustainability, 2012: 248-251.

[15] Yanlong Jiang, Weiwei Yuan, Hualin Li, Centralized cooling wind generator system, Patent application No.200610097464.4.

[16] Tianrong He, Heat pipe technology and its application in thermal engineering, Industrial Boilers, 2003, 78 (2): 24-27.

[17] David Reay, Peter Kew, Ryan Mc Glen. Heat Pipes (Theory, Design and Applications). Oxford: Elsevier Ltd, 2014: 66-67. 